\title{
On the Meaning of Effects of Substrate Structure on Biological Transport*
}

\author{
Halvor N. Christensen \\ The Department of Biological Chemistry, The University of Michigan \\ Ann Arbor, Michigan
}

No single approach or simple combination of approaches will bring us an understanding of membrane transport, how it occurs, how it is driven and how it drives other processes. The particularity of the minds of individual investigators usually ensures that numerous attacks on a problem are undertaken, and also that these attacks are continued, and reapplied even after other investigators may have begun to deplore them. This article was in the first instance initiated for myself, to rethink what it is that my associates and I hope to achieve by one set of approaches, namely by observing the results of modifying the structure of substrates on their transport. To a degree I am taking a precaution that we do not decide that such studies are obsolescent, that the approach is indirect, a "black box" and born of a lack of courage for direct isolative approaches, or that only superficial objectives can be pursued by the approach, simply because superficial objectives are so often pursued by it.

Perhaps it is enough to point out that even after isolation of a catalytic system or one of its components, much can be learned by looking for substrates that it handles in unusual ways. Consider how well this statement applies to chymotrypsin. No doubt it also applies to the leucine-binding protein and other binder proteins. Such isolated proteins may also be "black boxes" to direct inspection. Perhaps it is a fragmentary rather than a universal truth that the information one gets by isolation is so unique as to deserve sole attention. On the contrary, each step of isolation appears to increase the advantage with which other methods of study can be applied. Having begun my analysis, I will proceed, however, to try to illustrate what this approach can do by examples (one in some detail) of what it is now doing, a

* The experiments described here that derive from my laboratory, and the preparation of this manuscript, were supported in part by a grant (HDO1233) from the Institute of Child Health and Human Development, National Institutes, U.S. Public Health Service.

I speak also for my collaborators in these experiments, including M. E. Handlogten, A. M. Cullen, G. Ronquist and C. de Cespedes in the case of unpublished results.

Copyright (C) 1972 Plenum Publishing Company Limited. No part of this publication may be reproduced, stored in a retrieval system, or transmitted, in any form or by any means, electronic, mechanical, photocopying, microfilming, recording or otherwise, without written permission of Plenum Publishing Company Limited. 
procedure which can I suppose only vaguely reveal what it is capable of accomplishing.

1. To determine whether we are observing transport in the strict sense or instead a group translocation. Enzymologists bring to the transport field an apparently inexhaustible intuition that transport will prove to have as its basis a persisting enzymatic modification of the substrate. They also bring with them a firm understanding of how two reactions may be linked so that energy from one can be made available to drive another, but often a reluctance to accept a coupling whereby one of the two processes linked together is not a net reaction at all but a movement of a solute from one chemical potential to another-a coupling that we may call not chemical but chemiosmotic, if we allow ourselves to use that word in a broader sense than it is sometimes used. The transported sugar, the enzymologist may feel, will prove to have been mutarotated or phosphorylated, the transported amino acid will have been converted to an AMP anhydride or to an amide and so on. It is perhaps a parallel conservatism that wants to keep the coupling between electron transport and oxidative phosphorylation an organic chemical matter.

It was over three decades ago, we should remember, that three different $O$-methyl derivatives of glucose were shown to undergo typical intestinal transport, excluding the necessity of phosphorylation in either of three positions. ${ }^{1}$ It was also years ago that anhydroglucitol ("1-deoxyglucose") was shown to be transported in an apparently typical manner both by the intestine and by the human red blood cell, and a $\mathrm{Na}^{+}$-independent renal transport of 2-deoxyglucose has had recent extensive study. A new instance of inhibitory actions of these and other blocked sugars on the transport of galactose and 2-deoxyglucose have recently been reported for Aspergillus nidulans. ${ }^{2}$

To descend to a simpler and indeed an absurd level, it has been obvious from the first that $\mathrm{Na}^{+}$was transported without conversion to $\mathrm{K}^{+}, \mathrm{Ca}^{2+}$ without conversion to $\mathrm{Mg}^{2+}$, and so on. The student of inorganic-ion transport has therefore largely escaped this bias. Organic substrates of transport may also, however, be designed so they also escape modification. Amino acid analogs have been synthesized that undergo no sign of destabilization by the organisms and cells so far studied. Even distortion of the molecular structure seems largely excluded for some of these analogs, such as the one pictured in Fig. 1, and yet their transport is typical. ${ }^{3}$

It would be unwise at this point in history, however, to argue that metabolites are never transferred from one compartment of the cell to another by the process so lucidly described by Mitchell and Moyle a few years ago as group translocation, ${ }^{4}$ i.e., in the course of an enzymatic transfer of the chemical group from a donor to an acceptor. A membrane-associated phosphotransferase action on sugars is clearly of high metabolic importance in an as yet unknownumber of anaerobic 
bacterial species. It has been difficult to determine whether a phosphorylating enzyme actually exposes its reactive site on the external surface of the membrane to receive the sugar substrate, and then carries it through the membrane to bring it into contact with the phosphorylated heat-stable protein. The experiments of Gachelin indicate that it is probably another structure that binds the sugar during the translocation step, without changing the sugar into anything else, until it is brought into association with the phosphorylating system deeper in the cell. ${ }^{5}$ Nevertheless, the phosphorylation appears to be coupled to the mediated transport step, to transfer energy to it in an unexpected way. In addition it appears inherently likely that some simpler group translocations also take place, especially in subcellular metabolism.

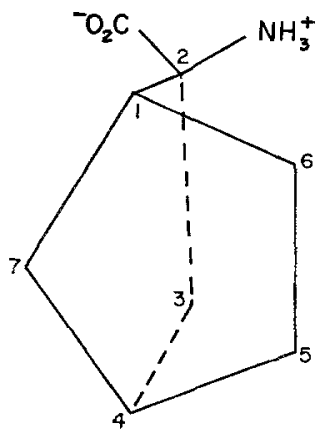

Figure 1. Structure of isomer of 2-aminobicyclo-[2,2,1]-heptane-2-carboxylic acid serving as a model substrate for transport.

If the positions of the amino and carboxyl groups are reversed one secures the exo-endo isomer to the structure shown. If that structural formula and the present one are viewed in a mirror, their stereoisomers will be pictured. Only the isomer shown undergoes mediated transport into $E$. coli $\mathrm{K} 12$ by System $L I V$, and only it stimulates release of insulin from the pancreatic islet. Furthermore this isomer corresponds more precisely than the others to the structural requirement of System $L$ of various cells. Hence the orientation of the pentagon seen in the foreground is critical, as is also the general location of the space requirement of the bicyclic structure with reference to the amino and carboxyl groups.

Although on paper one can arrange almost any enzymatic sequence to present a vectorial step, i.e., so that it provides a chemiosmotic coupling, it is striking, perhaps even embarrassing, how much more often the chemiosmotic coupling appears not to occur at the substrate level. Perhaps nature had first to solve the problems of the transport of electrons and inorganic ions. These solutions could then be extended to other transports by way of osmotic-osmotic coupling, thereby presumably reducing the evolutionary stimulus to development through subsequent modification of membrane structure of scores of totally new chemiosmotic couples in other transport systems. Even the phosphotransferase system appears to represent an energizing reaction, coupled to a molecular transport of the usual sort. ${ }^{5}$

Whatever the total role of group translocation, the point I should like to make here is not merely that molecular transport really also 
exists everywhere we look, but also how readily specific proposals of group translocation can be tested and excluded where they happen not to apply, by examining a range of analogs of the substrate that can be transported but some of which may be immune to the proposed chemical alteration. Too often one does not see these proposals challenged as vigorously as they readily could be. To a colleague who recently proposed an enzymatic cycle of group translocations as the basis for the transport of a class of amino acids, I offered this rather rash challenge: "I'll bet that you have on your own shelves the analog that will serve to exclude your proposal." Until one has tried hard to design analogs that are typically transported but do not undergo the metabolic modification supposedly inherent to the transport, and has thus dissociated the transport event as completely as possible from other events, one has not challenged well the relevance of that modification.

Another distinct possibility that can be eliminated or established by the study of variation of substrate structure is whether a metabolite must be converted into another substance before it can be accepted by the transport process. According to our experience, cystine appears to enter or leave some animal cells only after conversion to cysteine. In contrast the finding that cystine and diaminopimelic acid compete for the same transport system ${ }^{6}$ and for the same binding protein ${ }^{7}$ in Escherichia coli shows that cystine is undoubtedly transported by that cell as such. It is important to know for example to what extent glutamate might be transported after conversion to glutamine, or to what extent nucleosides and nucleotides may be interconverted either as a separate event before transport or as an inherent step in transport.

2. To determine whether we are observing transport by one, two or three or more systems. Perhaps I have already belabored sufficiently elsewhere the advantage of knowing which properties belong to the transport system we are attempting to describe, and which properties belong to another. ${ }^{8}$ I can make the new point that confusing relations will be observed between the properties of a binding protein and the transport activity with which it is presumed to be associated, if the latter is in reality produced by two transport systems. Furthermore, tests for the necessity for an energy input or for a co-substrate will yield confusingly complex results (e.g. indications of loose and variable coupling) if the contribution of a separate facilitated diffusion is unsuspected. We can expect to make little sense of the development of transport systems during embryonic life unless we differentiate among different systems.

In the meantime dozens of new instances of heterogeneity in the transport of a given metabolite have been reported. Hence we probably need say little more about the necessity of selecting or designing the substrate carefully if a single transport agency is to be studied in isolation, or about the confusion likely to arise when one is unknowingly studying the total activity of two or more processes at once. 
3. To describe in a complementary way the shape of a receptor site, i.e. the spatial distribution of the several components that recognize several chemical groups (usually three or more) in a configuration presented by effective substrates; also the space available and that not available around these components for other parts of the substrate molecule. I will consider first an example where no cosubstrate is recognized, and secondly cases in which the geometric description will include the relation between the positions taken by substrate and co-substrate. I shall begin by provisionally supposing the picture reached to represent a static site, nevertheless retaining for later development important reservations on that treatment.

The transport receptor site accommodating leucine, isoleucine and valine in $E$. coli K12 ("System LIV") provides space for the bulky structure of the bicycloheptyl ring of the norbornane amino acid shown in Fig. 1, but only if it lies in one of the four possible orientations with respect to the $\alpha$-amino and the $\alpha$-carboxyl group. ${ }^{3,9}$ The discrimination among these isomers could originate either from the spatial distribution of obstacles to the entry of the unsuitable substrates into the site, or from the distribution of structures providing favorable apolar bonding for the suitable substrates. Although reactivity with the amino acid transport systems usually increases with an increase in the number of methyl and methylene groups available for apolar bonding, all the isomers of the norbornane amino acid appear to provide several such groups at positions that produce reactivity for the branched-chain amino acids and their analogs. Hence it appears that the sharp rejection of three of the isomers is based on their encountering obstacles not met by the isomer shown in Fig. 1. Continuing tests of other unreactive and reactive analogs are refining the description of the receptor site. Apparently either this amino acid $^{3}$ or trifluoroleucine ${ }^{10}$ can be used to discriminate System LIV from a leucine-specific system in $E$. coli strains.

Let me offer next an example where both a substrate and a cosubstrate enter transport together. The experiments to be described were made for transport into the Ehrlich cell, the nucleated red cell of the pigeon, and the rabbit reticulocyte. The logic behind the exposition of this case began with observations for the transport system for cationic amino acids and progressed to findings for the neutral amino acid system $A S C$, for which the geometry of the receptor site can now be proposed in the greater detail. The basic amino acid System $L \dot{y}$ reacts strongly with amino acids carrying a cationic group on the sidechain. Unless that group tightly retains its proton, so that it presents a positive charge in an apparently apolar microenvironment at the transport site, the amino acid will show a high $K_{m}$ (i.e., poor reactivity) for System $L \dot{y}$; for example arginine and homoarginine are more reactive than lysine on that basis. Homoarginine is more reactive than arginine 
on another basis, presumably the added strength of the apolar bonding arising from its extra methylene group.

A number of unequivocally neutral amino acids at rather substantial concentrations are, paradoxically, able to inhibit transport of basic amino acids, or to enter countertransport with them. This activity has been found to depend strongly on the identity and concentration of the alkali-metal cation present in the suspending medium. Furthermore, the selectivity among the alkali-metal cations depends in turn on the structure of the amino acid. The neutral amino acids most effective in this reaction are also the ones whose action is most dependent on the

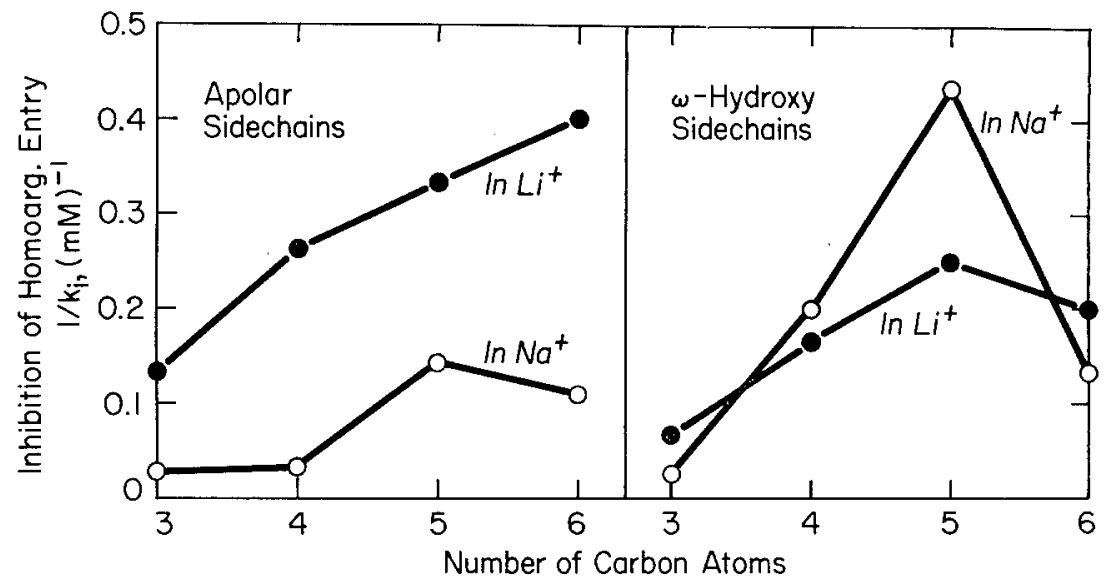

Figure 2. Effect of length of linear chains of amino acids on their inhibitory effect on homoarginine uptake by the Ehrlich cell, in the presence of $\mathrm{Na}^{+}$or $\mathrm{Li}^{+}$. Left: For amino acids with apolar sidechains, alanine to norleucine. Right: For $\omega$-hydroxy amino acids, from serine to $\epsilon$-hydroxynorleucine. $\left[\mathrm{Na}^{+}\right]$or $\left[\mathrm{Li}^{+}\right]=105 \mathrm{mN}$. Note the sharp reversal of the effectiveness of $\mathrm{Na}^{+}$and $\mathrm{Li}^{+}$when the hydroxyl group is on carbon five. $\mathrm{K}^{+}, \mathrm{Rb}^{+}$and $\mathrm{Cs}^{+}$ were also tested, but the inhibitory actions were very small when they replaced $\mathrm{Na}^{+}$in the modified Krebs-Ringer bicarbonate medium. Corresponding but somewhat different changes in selectivity were seen for the rabbit reticulocyte and in that case when the hydroxyl group fell on carbon four rather than on carbon five. Reproduced with permission from the Journal of Biological Chemistry. ${ }^{11}$

presence of a specific alkali-metal cation, namely $\mathrm{Na}^{+}$. To be at all inhibitory such amino acids must first of all meet certain structural requirements that we have come to associate with the cationic amino acid transport system (e.g. only one beta carbon; the amino group must be primary); but in addition for a strong interaction with a specific alkali-metal, a hydroxyl or mercapto group must be present on one specific carbon atom (carbon five, for the Ehrlich cell; carbon four, for the rabbit reticulocyte). Figure 2 illustrates the inversion of the preference between $\mathrm{Li}^{+}$and $\mathrm{Na}^{+}$as the hydroxyl group is shifted to carbon five for the former cell. The other three alkali-metal ions are very low in enhancing activity in that cell, ${ }^{11}$ and hence are not represented in Fig. 2. 
We suppose the neutral amino acids that interact strongly with a specific alkali-metal ion to inhibit transport by the cationic amino acid system are the ones that first of all can enter Site $L \dot{y}$ even if they do not occupy it completely. We suppose furthermore that these neutral amino acids become bonded to the inorganic ion when both substrates are bound at the receptor site, since all those identified as highly reactive at this time contain a sulfur or an oxygen atom on the sidechain and because the interaction is so sensitive to the position of that oxygen or sulfur atom. We suppose that the alkali-metal ion then occupies the position at the receptor site otherwise taken by the positively charged, distal nitrogenous group of the basic amino acid. ${ }^{11-13}$

We were led then to ask whether the converse inhibitory reaction also occurs: Does arginine inhibit the linked transport of neutral amino acids plus $\mathrm{Na}^{+}$by systems primarily reactive with neutral amino acids? An apparently ubiquitous system for straight-chain, neutral amino acids, System $A S C$, was studied in the rabbit reticulocyte and the pigeon red blood cells. Its typical substrates are alanine, serine and cysteine (hence the abbreviated designation $A S C$ ) and their 4- and 5 -carbon homologs. In both cases arginine was inhibitory to serine uptake, although in this case no exchange of arginine for $\left(\mathrm{Na}^{+}+\right.$serine $)$ across the plasma membrane could be detected. Either the guanidinium, methylguanidinium or lithium ions was inhibitory to transport by competition with the co-substrate, $\mathrm{Na}^{+} .{ }^{14}$

These inhibitory effects led us to study more closely the normal interaction between the two co-substrates, amino acid and $\mathrm{Na}^{+}$, in System $A S C$. Both of these co-substrates must be present on both sides of the membrane for transport to take place in nucleated and reticulated red cells. The transport involves the exchange of a neutral amino acid plus $\mathrm{Na}^{+}$for either the same or a different neutral amino acid plus $\mathrm{Na}^{+}$, although the number of sodium ions moving in each direction is not necessarily equal when the two amino acids are dissimilar. As the concentration of each co-substrate was raised, the apparent $K_{m}$ of the other co-substrate fell. This effect ranged from very small for proline, to very large for the amino acids with a hydroxyl or sulfhydryl group on carbon three or four. Furthermore, the stoichiometry of the flux augmentations rose sharply as the hydroxyl group was placed in the optimal position. About five proline molecules entered the cell with each sodium ion, whereas about three sodium ions were taken up with each serine molecule, and about four sodium ions with each cysteine molecule. These results show that the interaction with $\mathrm{Na}^{+}$was again strongly influenced by the presence of an $\mathrm{O}$ or $\mathrm{S}$ atom in a particular position. Furthermore, the preferred position was distinctly different for this system than for the cationic amino acid transport system. Other evidence is also overwhelmingly against the idea that System $A S C$ and the cationic amino acid system are merely two manifestations of 
the same entity, the $K_{i}$ values being totally inconsistent with the idea. ${ }^{13}$ Furthermore, the cationic amino acid system persists on maturation of the reticulocyte after we no longer can detect System $A S C .{ }^{15}$

Finally, the orientation of the hydroxyl group at the preferred position on carbon three or four, whether cis or trans to the carboxyl group of the amino acid, is decisive to the interaction with $\mathrm{Na}^{+}$in System $A S C$. If a cis hydroxyl group is introduced into proline, the effects on transport and on interaction with $\mathrm{Na}^{+}$are not favorable. If instead the hydroxyl group is trans, both transport and potentiation of transport by $\mathrm{Na}^{+}$are large, and the flux stoichiometry, $\Delta \nu_{\mathrm{Na}^{+}} / \Delta \nu_{\mathrm{aa}}$, is sharply

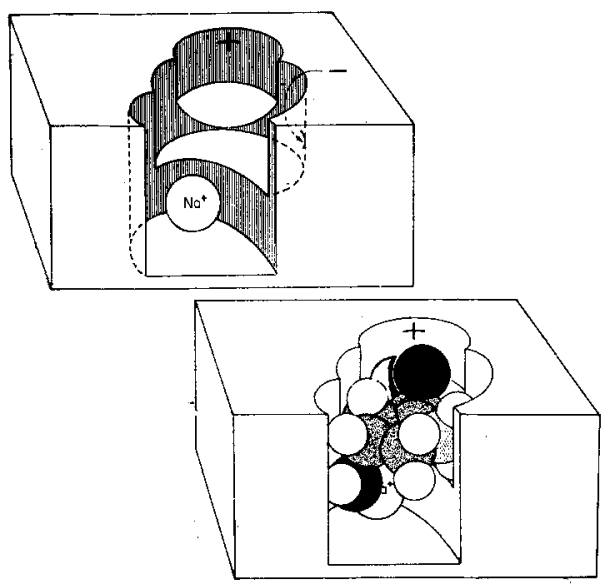

Figure 3. Diagrammatic representation proposed for transport site ASC, showing $\mathrm{Na}^{+}$ (top) and both $\mathrm{Na}^{+}$and hydroxyproline (bottom) in place.

The arrow points to at structure that recognizes the protonated $\alpha$-amino or $\alpha$-imino group; the plus sign identifies a structure recognizing the carboxylate group. Undoubtedly the receptor structure to some degree mediates the interaction between the two cosubstrates. Structural considerations persuade us, however, to propose a direct interaction between the two, as suggested by the lower sketch. No implication is intended that $\mathrm{Na}^{+}$must enter the site first. See text for further discussion.

increased. ${ }^{16}$ Because the aminoprolines are not admitted to site $L y$ in the cells we have so far studied, we have not yet attained an unequivocal determination of the corresponding geometric feature of that site.

These results lead us to propose geometric relations in the positions taken by the amino acid and $\mathrm{Na}^{+}$at receptor Site $A S C$ resembling those crudely pictured in Fig. 3. The $\mathrm{Na}^{+}$is visualized as lying nearer to carbon four than to carbon three, at a distance such that bonding can occur to an oxygen or sulfur atom attached to one of these carbon atoms. Furthermore the position of $\mathrm{Na}^{+}$is pictured as trans to the structural feature of the site that determines the position taken by the carboxyl group. We are led also to believe that apolar bonding occurs to the cis aspect of the methyl or methylene group represented by carbon three, 
because of the especially unfavorable effect of a 3-cis hydroxyl group. ${ }^{17}$ The unusually high stereospecificity of System $A S C$ gains added interest when we propose a different kind of bonding, in addition to apolar bonding, to the amino acid sidechain. The new kind of bonding to the site must be absolutely unavailable to the sidechain if it takes the position characteristic of $\mathrm{D}$-amino acids, whereas the needed apolar bonding must be more diffusely available.

Information of this kind shows the word co-transport has turned out to be more precise than it might have proved: The co-substrates appear to bind in juxtaposition and to interact with each other. Until we describe this aspect of their behavior we will not be prepared to understand why each is necessary for the transport of the other.

Reference should also be made to the classical demonstration by Le Fevre and Marshall that the affinity of various monosaccharides for uptake by the human red blood cell is determined by the number of hydroxyl groups offered in an equatorial arrangement by the more stable conformer of the sugar. ${ }^{18}$ I suspect for certain $\mathrm{Na}^{+}$-dependent sugar transport systems that one or more of the hydroxyl groups of the sugar (possibly that on $\mathrm{C} 2$, because it is most decisive to $\mathrm{Na}^{+}$-dependent transports) may bond to $\mathrm{Na}^{+}$. Several 2-deoxy sugars are taken up by kidney cells by a $\mathrm{Na}^{+}$-independent transport system, and not by the usual $\mathrm{Na}^{+}$-requiring system of that tissue, ${ }^{19}$ a contrast that may also point to the 2-hydroxyl group.

4. To modify the selectivity among analogous co-substrates. In the preceding section we found ourselves fortuitously already pursuing this goal: As we change the structure of the neutral amino acid, the relative effectiveness of $\mathrm{Na}^{+}, \mathrm{Li}^{+}$and $\mathrm{K}^{+}$as co-substrates in the reaction with System $L y$ changes (see for example Fig. 2). We are following the same approach with System $A$ to see whether we can tell whether the $\mathrm{Na}^{+}$ lies near any particular part of the amino acid molecule at that receptor site. In this case the structural features that intensify the preference of $\mathrm{Na}^{+}$over $\mathrm{Li}^{+}$are giving us very promising signals of nearness of the two co-substrates although the topography is unlike that for System $A S C$.

5. To discriminate sequential events in transport and to determine the nature of each.

(a) By design of substrates not entering steps subsequent to binding. In the preceding section we have largely assumed that our experimental results were describing for us a static receptor site. In actuality it is already possible to see that successive chemical events are occurring. For example, $\mathrm{Li}^{+}$plus a neutral amino acid substrate can occupy Site $A S C$; either arginine or methionine ${ }^{14,20}$ can occupy the site without kinetic evidence that $\mathrm{Na}^{+}$is binding also. In each of these three cases, however, transport does not occur. Hence both co-substrates must have the right structure; otherwise the site fills but transport does not occur. The opportunity arises to discover the nature of the event that follows 
$\mathrm{Na}^{+}$binding, but not $\mathrm{Li}^{+}$binding, when these ions enter the site in juxtaposition to the amino acid substrate.

Caspary, Stevenson and $\mathrm{Crane}^{21}$ have observed an opposite kind of case: 6-deoxy-L-galactose ( $\mathrm{L}$-fucose) shows a $\mathrm{Na}^{+}$-dependent inhibition of glucose transport by the intestine. In this case even though the alkali-metal ion is the right one so that occupation of the site can presumably be completed, the monosaccharide is still structurally unsuitable for a subsequent event.

Researches on transport frequently stop short of an important opportunity when they merely show that an analog is inhibitory to the uptake of a standard substrate for a given transport system. The roles of substrate and inhibitor should be reversed, to see if the response of the receptor site to the inhibiting analog is complete transport. It becomes a more remarkable case when l-anhydroglucitol is a substrate as well as an inhibitor of galactose transport. On the other hand a valuable investigative opportunity is uncovered if anhydroglucitol should prove instead to be only an inhibitor and not a substrate, because one could then explore the relation of the failure to the missing l-hydroxyl group. The several opportunities mentioned above have not been well exploited yet, although we can begin to see how they can be used. One must of course think chemically about the differences between competing analogs. The experience described in this section counsels us, whenever we see evidence that two substrates of distinctly different charge (e.g. aspartate and fumarate) are competing for the same receptor site, to look for an inorganic ion co-substrate as the equalizing factor. Where one sodium ion is co-substrate to the ordinary neutral amino acids for transport, it is perhaps plausible that two sodium ions, even though in a different transport system, might be co-substrate to aspartate or glutamate. ${ }^{22}$

(b) Design of substrates that slow a subsequent step, both for uptake and entry. Other modifications of transport behavior by variations in substrate structure may also be informative. We have noticed, for example, that all the ordinary substrates of System $A$ of the Ehrlich cell show about the same $V_{\max }$ for uptake, namely about 5 or $6 \mathrm{mmol}$ per $\mathrm{kg}$ cell water'min. But $\alpha$-(methylamino)-isobutyric acid shows a $V_{\max }$ of about one-third this value. ${ }^{23}$ Apparently the structural features of the ordinary amino acids do not "obtrude" to change the rate of the rate-limiting event, but the $N$-methyl group does in this case obtrude. We have been able to take advantage of its slowing of the translocative event by demonstrating the phenomenon of trans inhibition with this amino acid (Fig. 4)..$^{24}$ It had been predicted by Heinz and Durbin that trans stimulation would be replaced by trans inhibition if the rate of reorientation of the filled site could be changed from greater than that of the empty site, to a rate lower than that of the empty site. ${ }^{25}$ Figure 4 shows that external AIB causes an acceleration of the exodus of either 
AIB or MeAIB from the Ehrlich cell, whereas MeAIB causes a slowing of the exodus of either test amino acid. Hence the structure of the amino acid that drives the trans effect rather than the one that records it apparently is decisive as to whether the effect is positive or negative. Trans inhibition by MeAIB occurs equally well for uptake and for entry (see Fig. 6 in ref. 24); i.e. no evidence is obtained for an asymmetric action of the $N$-methyl group on translocation. Appearance of the predicted trans inhibition provides valuable support for the approximate correctness

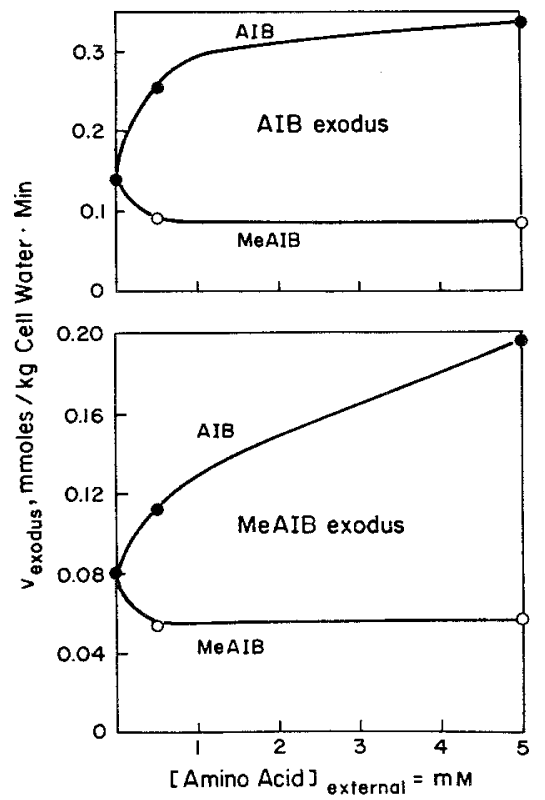

Figure 4. Contrasting production of trans stimulation of amino acid exodus from the Ehrlich cell by external $\alpha$-aminoisobutyric acid, and trans inhibition of exodus by its $N$-methyl derivative.

The labeled amino acid as indicated had first been introduced into the cell at an estimated mean internal concentration of about 1.4 mmolar. The loss of the label was then observed during two min into Krebs-Ringer medium containing the indicated concentration of AIB or MeAIB. Note that the responses of labeled AIB and MeAIB are essentially the same; i.e., the results are uninfluenced whether homo- or hetero-exchange is being observed.

of aspects of the model for transport under which we are proceeding. In addition it offers some as yet unclear opportunities to discover something about the nature of the translocation event: Why does the $N$-methyl group obtrude to cause slowing when the 5-methylene group of proline does not?

This kind of trans inhibition lowers both of the two opposed fluxes and does not apparently modify the equilibrium position finally reached; but presumably it can slow progress of net uptake to such a degree that in the real case uptake effectively may stop far short of equilibrium, as appears to occur in Neurospora. ${ }^{26}$ Such an arrangement could prevent 
over-charging of the amino acid pool of a cell, and for a tightly-coupled uphill transport should conserve energy. It should, however, leave the organism sensitive to an excess of any single substrate of the system concerned, because the uptake of other substrates of the same system would presumably also be interrupted.

Several years ago, Oxender and Whitmore ${ }^{27}$ showed that a 2-min treatment of the Ehrlich cell with 0.5 mmolar 2,4-dinitrofluorobenzene minimizes or abolishes trans stimulation by substrates of transport System $L$, without causing a proportional slowing or diminution of their net uptake. This independent abolition of exchange seemed to indicate that exchange and net uptake occur by different components of the transport System $L .{ }^{27}$ But is it not more likely that trans stimulation is eliminated by a specific slowing of the reorientation of the substratecarrier complex just as it was through the methylation of AIB? In testing this hypothesis we have shown that treatment of the Ehrlich cell with DNFB slows not only uptake of 2-aminobicycloheptane-2carboxylic acid by amino acid-depleted cells, but also its exodus into amino acid-free medium, i.e. net exodus. So far the only way we have to show that this slowing does not apply also to reorientation of the unloaded as well as the loaded carrier is the near elimination of trans stimulation, not unfortunately an independent proof. If we select one of the more slowly migrating substrates of System $L$, DNFB may be able convincingly to replace even the powerful trans stimulation of System $L$ with trans inhibition.

A fundamental question inherent in these tests is whether one can indeed eliminate trans stimulation (accelerative exchange) or trans inhibition by changing substrate or membrane structure in any way other than by manipulation of the relative rates of migration by steps inherent to the transport process itself.

(c) Design of substrates that accelerate a subsequent step. An effect of this kind has presumably been encountered in the study of System $A S C$ already reported. The presence of a properly orientated hydroxyl or mercapto group appears to accelerate differentially the release of $\mathrm{Na}^{+}$ from the ternary complex, thereby sharply increasing the coupling stoichiometry of $\mathrm{Na}^{+}$to amino acid. ${ }^{16}$

Acceleration of the translocation step appears to be produced in a number of cases by the presence of a second amino group, usually on the $\gamma$-carbon atom. Oddly enough, this behavior is seen with systems for neutral amino acids, for which diamino acids would not be expected to serve as substrates. The first such case encountered was $\alpha, \gamma$-diaminobutyric acid, which shows a remarkably high $V_{\max }$ for System $A$ of the Ehrlich cell, over $60 \mathrm{mmol}$ per $\mathrm{kg}$ cell water $\cdot \min$. Since $\mathrm{p} K_{2}^{\prime}=8 \cdot 4$, this compound is actually cationic to the extent of about $91 \%$ in free solution at $\mathrm{pH} 7 \cdot 4$. A corresponding transport by System $L \dot{y}$ can easily be measured. Apparently at the transport receptor Site $A$, however, 
it is stabilized in the form in which the $\gamma$-amino acid group is not protonated and hence the compound also enters transport as a neutral $\alpha$-amino acid by that system. Such a stabilization could well result if the $\gamma$-group falls in a highly apolar region at the transport receptor site.* We will consider later the question why the $V_{\max }$ of this amino acid should be so high.

Here we presume the hydrogen ion, rather than an alkali-metal cation, serves as the factor compensating for the difference in charge among competing substrates, a circumstance that could permit energy transfer from a movement of hydrogen ion, down a gradient. This possibility recalls the apparent service of $\mathrm{H}^{+}$, in place of $\mathrm{Na}^{+}$as a co-substrate for uptake of neutral amino acids by yeast. ${ }^{28}$

Note that the chemical form in which we believe $\alpha, \gamma$-diaminobutyric acid to be stabilized on its entry into transport System $A$, namely

$$
\mathrm{H}_{2} \mathrm{NCH}_{2} \mathrm{CH}_{2} \mathrm{CH}\left(\mathrm{NH}_{3}^{+}\right) \mathrm{COO}^{-}
$$

should be an excellent substrate also for System $A S C$, just as the closely analogous homoserine is. Instead the diamino acid is essentially inert with respect to System $A S C$; a $50-\mathrm{mm}$ concentration shows less than a $4 \%$ effect on the uptake of $\mathrm{L}$-serine by the pigeon red blood cell or the

TABLE I. Predicted route of transport of diamino acids

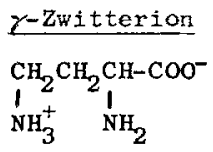

B system?

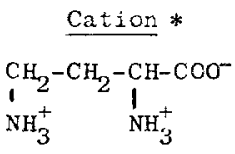

$\underline{\mathbf{L}} \stackrel{+}{\mathrm{y}}$ system
$\alpha-Z w i t t e r i o n$<smiles>NCCC([NH3+])C(=O)[O-]</smiles>

ASC and A systems

Found:

partial inhibition by B-alanine partial inhibition by lysine
All criteria for powerful uptake by System A; Negligible uptake by System ASC

Conclusion: This amino acid reacts like an $\alpha$-Zwitterion with System $\underline{A}$, but with System ASC it does not:

\footnotetext{
* Note the inductive action between the two amino groups of this compound. The cationic form shown in the upper middle of this table, and also that shown in a similar position in Table III, can dissociate in either of two ways : to form an $\alpha, \gamma$-dipolar ion as shown at the left, or to form an $\alpha, \alpha$-dipolar ion as shown at the right. For diaminobutyric acid, also for 4-aminopiperidine-4-carboxylic acid, as shown in Table III, and for several other of the basic amino acid discussed here, either of these pathways may occur rather readily and the proportion dissociating in a given way should be sharply modifiable according to the environment the two amino groups find at the receptor site. Once one of these protons has been lost, however, the other comes by an inductive effect to be more strongly retained. Conversely, stabilization of the retention of one of them causes the other to be lost more readily. If we guanylate the piperidine $N$ (lower, central structure in Table III), the resulting guanidinium group will not readily lose its positive charge. Hence the dissociation will be stimulated to proceed along the pathway toward the lower left, to form an $\alpha, \gamma$-dipolar ion, which is not a substrate for any of the transport systems under consideration.
} 
rabbit reticulocyte. This finding is not paradoxical when we remember that the $\gamma$-amino group will find no apolar region at the $A S C$ receptor site, if the model suggested in Fig. 3 is correct. Nevertheless the contrasting acceptance of this amino acid by System $A$ and its rejection by System $A S C$ tells us of an important difference in these two. Table I presents the contrasting behavior of $\alpha, \gamma$-diaminobutyric acid just described.

Other cases in which the state of dissociation of a basic amino acid appears to be modified in the environment intrinsic to transport have been encountered for several lysine analogs and will now be discussed at considerable length. Lysine itself was found surprisingly to behave

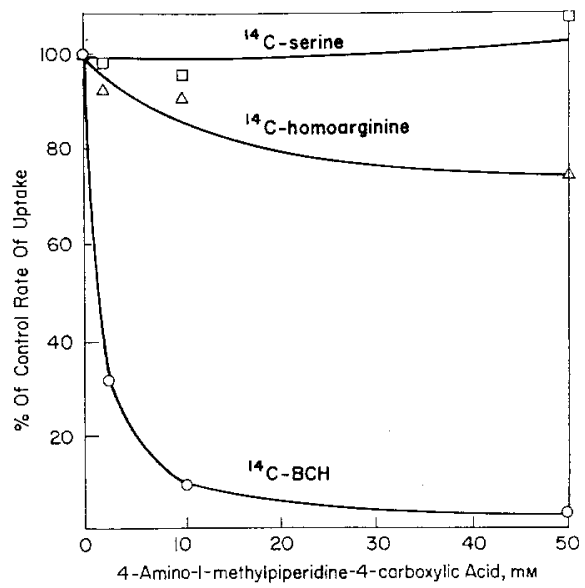

Figure 5. Inhibition by 4-amino-1-methylpiperidine-4-carboxylic acid of uptake of three labeled amino acids by the Ehrlich cell.

Uptake of the test amino acid measured during $1 \mathrm{~min}$ at $37^{\circ}$ and $\mathrm{pH} 7.4$ from KrebsRinger bicarbonate medium. The failure to inhibit serine uptake reveals negligible reaction with Systems $A$ and $A S C$. The action on homoarginine uptake shows weak reaction as a cationic amino acid, whereas the inhibition of BCH uptake shows a strong reaction with System $L$.

to a considerable extent as a neutral amino acid, presumably through a degree of repression of the same sort of the protonation of its epsilon amino group. ${ }^{13}$ For that reason it cannot be trusted as a test substrate specific to the cationic amino acid transport system. Instead arginine analogs, which are unambiguously cationic, must be recommended as the model substrates for System $L \ddot{y}$.

Figure 5 illustrates one of the methods we used for investigating transport properties of the lysine analogs.* A piperidine amino acid shows itself ineffective in inhibiting serine uptake by the Ehrlich cell during $1 \mathrm{~min}$ (upper curve), effective only at high levels in inhibiting

* The results of Tables II and III and Figs. 5 to 9 have been reported in a preliminary form. ${ }^{29}$ M. E. Handlogten and A. M. Cullen collaborated with the author in obtaining the results to be described by these tables and figures. 
homoarginine uptake (middle curve), but extremely effective in inhibiting uptake of the norbornane amino acid that serves as a model substrate for System $L$, the system for neutral amino acids with bulky, apolar sidechains (lower curve). Some of the analogs tested (Table II) are either piperidine amino acids (the first three) or 4-aminocyclohexyl amino acids (the last two). Because their $\mathrm{p} K_{2}^{\prime}$ values lie between $\mathrm{pH} 7$ and 8 , they should be present in free solution partly as cations and

TABLE II. Transport reactivity of analogs of basic amino acids in the Ehrlich cell

\begin{tabular}{lccc}
\hline & $\begin{array}{c}K_{i} \text { on } \\
\text { homoarginine } \\
\text { uptake } \\
(\mathrm{mM})\end{array}$ & $\begin{array}{c}K_{i} \text { on uptake } \\
\text { of norbornane } \\
\text { amino acid } \\
(\mathrm{mM})\end{array}$ & $\begin{array}{c}\text { Distr. ratio } \\
\text { at } 60 \text { min }\end{array}$ \\
\hline $\begin{array}{c}\text { 4-Aminopiperidine- } \\
\text { 4-carboxylic acid }\end{array}$ & 50 & 15 & $12 \cdot 8$ \\
$\begin{array}{l}\text { 1-methyl derivative } \\
\text { 1-guanyl derivative }\end{array}$ & 90 & 1 & 25 \\
$\begin{array}{c}\text { Cis-1,4-Diaminocyclo- } \\
\text { hexanecarboxylic acid* }\end{array}$ & 30 & $>100$ & 12.7 \\
Trans isomer* & 60 & $2 \cdot 7$ & 10.2 \\
\hline
\end{tabular}

* The cis isomer was separated from admixture with the trans isomer by conversion to the copper salts, the cis isomer forming a particularly insoluble salt. Structure was established by showing that diamino acid forming the soluble copper salt can be cyclized to an $\alpha$ piperidone, as ornithine can, by neutralizing its methyl ester hydrochloride with excess freshly precipitated hydrous silver oxide, ${ }^{30}$ whereas the diamino acid isolated as the insoluble copper salt cannot. Synthesis of the mixture of isomers proceeded via 4-toluene-sulfamidocyclohexanone, converted to its aminonitrile by the Strecker synthesis, and the latter to the free amino acid by $\mathrm{HCl}$ hydrolysis. The Bucherer-Libe synthesis yielded mainly the trans isomer.

partly as dipolar ions, the latter probably largely $\omega$-zwitterions. All of them react extremely weakly with the transport system for cationic amino acids (first column of data) unless an unambiguously cationic group is produced either by dimethylation or by guanylation of the distal $\mathrm{N}$ atom (third compound listed).

The simplest, unsubstituted piperidine amino acid (the first compound listed) proves to be a fairly good substrate for the transport System $L$ for neutral amino acids (middle column of data). Hence here also each of the receptor sites of two transport systems appears to suppress the otherwise likely protonation of the terminal cationic group. This interpretation is strengthened by the effect of methylation of the piperidine $\mathrm{N}$ (second line) - the product is fifteen times as reactive with the neutral System $L$, and now it is even less reactive than before as a cationic amino acid. Presumably this methyl group lowers only moderately the $\mathrm{p} K^{\prime}$ of the piperidine $\mathrm{N}$, its main effect perhaps being 
to assist the accommodation of the unprotonated sidechain into the apolar microenvironment supplied for the sidechain at Site $L$.

Similar behavior is seen for what we may call the next higher homo$\log$, 1,4-diaminocyclohexanecarboxylic acid, when the two amino groups are in the cis relation. It is highly reactive with System $L$, very weakly with System $L \dot{y}$. If, however, the two amino groups are in the trans orientation, the transport reactivity for System $L$ is substantially weaker. It should be noted also that the several amino acids mentioned so far are concentrated 10- to 25-fold by the Ehrlich cell, except that the trans diamino acid (lower right) is much less strongly concentrated.

Does this cis, trans difference serve to report to us differences in the microenvironment encountered by the two sides of the amino acid sidechain? Perhaps, but not necessarily. We may instead suppose that the cis isomer has the advantage that its $\delta$-amino group as it enters the apolar environment is able to donate its proton directly to the $\alpha$-amino group, whereas for the trans isomer no such approach and donation can take place. To discriminate between these possibilities we need lysine analogs that present separately each of these two features, namely (1) a cis orientation of the two amino groups, and (2) approachability of the two amino groups. For the present, then, we cannot draw an unequivocal 3-dimensional picture for Site $L \hat{y}$.

The poor reactivity of these diamino acids as cationic substrates is unequivocably contrary to the prediction from their titration curves. In free solution they are certainly present from 25 to $50 \%$ as cations (39\% in the case of the $N$-methyl derivative), but their low reactivity shows that they maintain no such structure at the $L y$ site. Furthermore, from the reactivity of the corresponding $N$-dimethyl and guanyl derivatives, we know there is no lack of room at the site for amino acids with the $N$-methylpiperidine or aminocyclohexane structure. Hence we suppose that the recognition site of System $L \dot{y}$, as well as that of $L$, presents an environment unfavorable to the retention of a proton on the distal amino group.

We will return then to the reaction of diamino acids with Site $L$. Figure 6 shows that the species of 4-amino-1-methylpiperidine-4carboxylic acid reactive with System $L$ appears as expected to be the dipolar ion $\mathrm{MPAA}^{ \pm}$. As the $\mathrm{pH}$ is raised, the concentration of the transport reactive species may be seen to increase in approximate proportion to the amount of the dipolar ions generated. But that result does not tell us which dipolar ion, the $\alpha$-or the $\gamma$-zwitterion. For the 1-methylpiperidine amino acid, $\mathrm{p} K_{2}^{\prime}$ is $7 \cdot 2$ and $\mathrm{p} K_{3}^{\prime}, 9 \cdot 7$. The question is, which group does $\mathrm{p} K_{2}^{\prime}$ represent, and which group does $\mathrm{p} K_{3}^{\prime}$ represent? We can readily answer that question for the benzyl amino acid, for which $\mathrm{p} K_{2}^{\prime}=6.9$ and $\mathrm{p} K_{3}^{\prime}=9.2$ (Fig. 7). Figure 8 shows at the left the change in the UV spectrum during its titration. As the two steps $\mathrm{p} K_{2}^{\prime}$ and $\mathrm{p} K_{3}^{\prime}$ of the titration curve are produced, the UV absorption may be seen to 


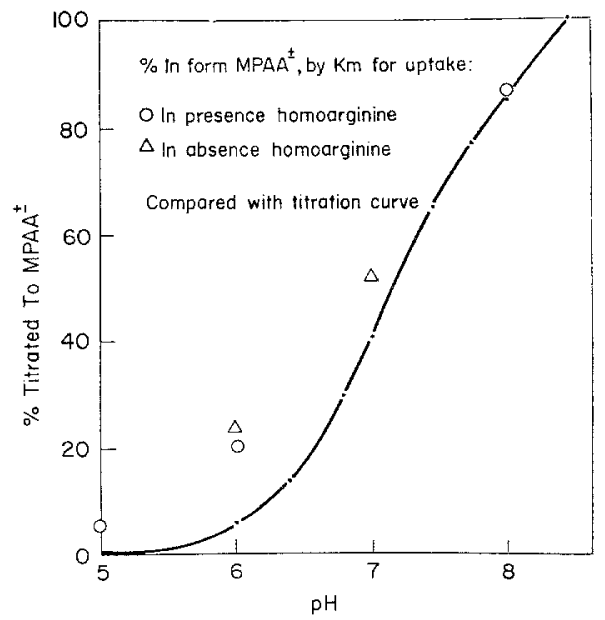

Figure 6. Identification of an isoionic form as the species of 4-amino-1-methy]-piperidine4-carboxylic acid reactive with transport System $L$.

The solid line is a portion of the titration curve of the amino acid at $25^{\circ}, \Gamma / 2=0 \cdot 1$, and shows the proportion present in the isoionic form $\mathrm{MPAA}^{ \pm}$as a function of $\mathrm{pH}$. The circles and triangles show the proportional amount present in the transport-reactive species as indicated by the measure of $K_{m}$ for uptake at $37^{\circ}$, the triangles representing total uptake, and the circles, the uptake retained in the presence of $20 \mathrm{~mm}$ homoarginine. Krebs-Ringer phosphate medium adjusted to the indicated $\mathrm{pH}$ values, under an oxygen atmosphere. "This result does not tell us however which isoionic form, the $\alpha$ - or the $\gamma$-zwitterion, is the reactive species. The demonstration is precisely valid only if System $L$ is $\mathrm{pH}$-independent-which as we shall see is not quite the case.

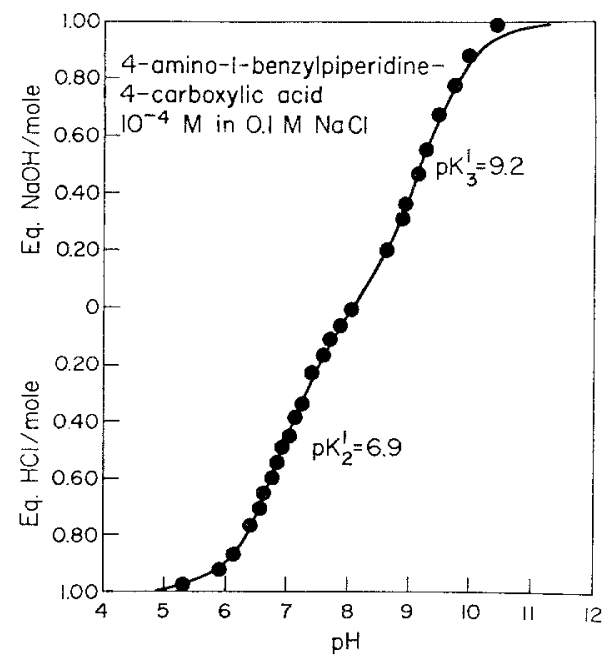

Figure 7. Titration of 4-amino-1-benzylpiperidine-4-carboxylic acid with $\mathrm{NaOH}$ and $\mathrm{HCl}$.

The amino acid was $10^{-4} \mathrm{M}$ in $0.1 \mathrm{~N} \mathrm{NaCl}$ at $25^{\circ} \mathrm{C}$. This result does not tell us of course to what degree the $\alpha$-and the $\gamma$-nitrogen atoms figure in each of the two titration steps.

increase sharply at wavelengths of about 262 and 268 nanometers, producing the two peaks seen in the middle of the figure. Of the total increase in absorbancy at these peaks, $41 \%$ occurs in association with $\mathrm{p} K_{2}^{\prime}$ (note separation of the lowest and the middle curve) and $59 \%$ 
occurs in association with $\mathrm{p} K_{3}^{\prime}$ (note separation of the middle and the highest curve).

For reference purposes we made the same titration with 1-benzyl-4piperidone which has only a single stage of dissociation since it has no other titratable group than the piperidine N. The same development of the two peaks now takes place in the single step. This result identifies

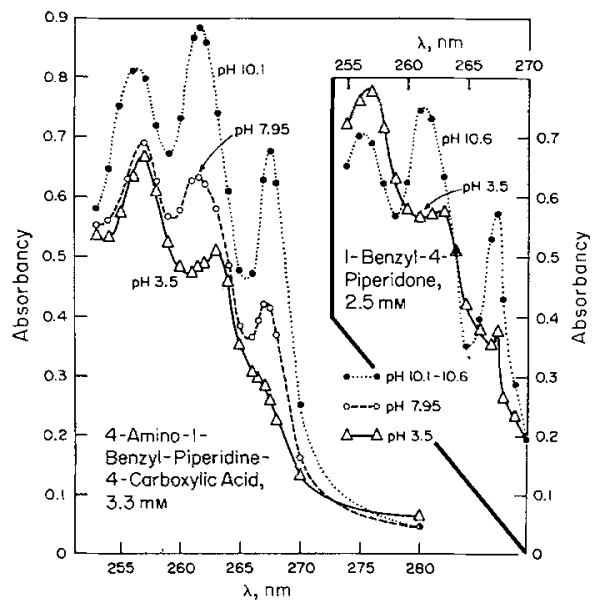

Figure 8. Change of ultraviolet spectrum of 4-amino-1-benzylpiperidine-4-carboxylic acid, $3.3 \mathrm{~mm}$, and of 1-benzyl-4-piperidone, $2.5 \mathrm{~mm}$, with change of $\mathrm{pH}$.

$\mathrm{NaCl}=0.1 \mathrm{~N}, 25^{\circ} \mathrm{C}$. The indicated $\mathrm{pH}$ values were obtained by adding very small volumes of $\mathrm{HCl}$ or $\mathrm{NaOH}$. (See text for discussion.)

the appearance of these two peaks with the formation of the unprotonated benzylpiperidine group. Accordingly, the two upper steps of the titration of the benzylpiperidine amino acid are severely hydridized, the first step involving $41 \%$ protonation of the piperidine $\mathrm{N}$ and $59 \%$ protonation of the $\alpha$-amino group. The second step then completes both protonations.

Our use of the benzyl group, however, undoubtedly directs the initial protonation toward the piperidine $N$, in comparison with the 1 -methyl analog. For example the $\mathrm{p} K^{\prime}$ of 1-benzylpiperidone is $7 \cdot 2$, whereas that of 1-methyl-4-piperidone is $8 \cdot 0$. Therefore the latter amino acid will certainly form less $\alpha$-zwitterion than the former in free solution. Note that this difference of 0.8 is the same we saw between the 1-benzylpiperidine and 1-methylpiperidine amino acids; but in that case the difference was divided between the two groups in the ratio $3: 5$. This result tells us that $\mathrm{p} K_{3}^{\prime}$ of the 1-methylpiperidine amino acid is associated to a larger extent with the piperidine $\mathrm{N}$ than with the $\alpha$-amino group, since that nitrogen atom will certainly be the more sensitive to our substitution of the benzyl group for the methyl group. From the relation of these $\mathrm{p} K^{\prime}$ values it can be estimated that at least 5 molecules of the $\delta$-zwitterion should be present at $\mathrm{pH} 7.4$ for each 
molecule of the $\alpha$-zwitterion. This calculation means that we should divide by 6 the $K_{i}$ of the methylpiperidine amino acid describing its reactivity with System $L$, in order to obtain the value of $K_{i}$ applying to the $\alpha$-zwitterionic species. The value of $0.17 \mathrm{~mm}$ thus obtained is unrealistically low in comparison with the values shown by analogs certainly not present as the $\delta$-zwitterion, e.g. the 1 -formylpiperidine amino acid or 1-aminocyclohexane-1-carboxylic acid. Accordingly we conclude that the $L$ site has stabilized the methylpiperidine amino acid in the $\alpha$-zwitterionic form, thus producing an unexpected reactivity with System $L$, far beyond that to be expected from the quantity present in that form in free solution.

\section{TABLE III}

Predicted Route of Transport of Diamino Acids

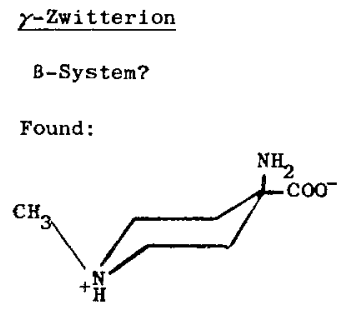

\section{Cation}

$\underline{\alpha-Z w i t t e r i o n}$

$$
\text { System } \stackrel{+}{\underline{L}}
$$

System $\underline{L}$ or $\underline{A}$

Found:
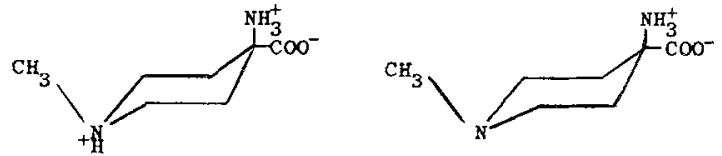

Not tested

Little if any

(mutual inhibition

with homoarginine,

max. Iess than $15 \%$

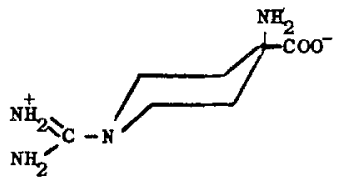

Not tested

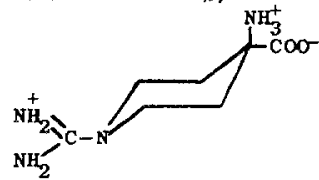

Strong with $\underline{L}\left(K_{m}=0.5 \mathrm{mH}\right)$

(Typical competition with $\mathrm{BCH})$

(Statistically improbable form)

Conclusion:

Strong $\left(K_{m}=2.3 \mathrm{mM}\right)$

(Typical competition

with homoarginine)

Litle if any (tutual

inhibition with $\mathrm{BCH}$

max. less than 16\%)

4-amino-1-methylpiperidine-4-carboxylic acid behaves

unexpectedly as an $\alpha-Z w i t$ terion with respect to system $\underline{L}$

Table III summarizes the contrast between the 1-methyl and the 1 -guanyl piperidine amino acid. The first behaves as a neutral amino acid with respect to System $L$, even though only a small part is present in the reactive form in free solution. In the second case, guanylation of the piperidine $\mathrm{N}$ has produced so high an affinity for the hydrogen ion that it reacts for transport only as a cationic amino acid.

We should emphasize that we are by no means proposing that the production of an uncharged sidechain is necessary to permit entry of these hydrophilic substrates into the bulk apolar environment of a lipid pool, e.g. that presented by a bimolecular lipid bilayer. Clearly certain 
parts of the amino acid molecule encounter instead polar areas at which they are bound, and only a limited and distinct part of the molecule encounters apolar bonding. I question whether there is good evidence for an indiscriminate conversion of amino acids or sugars into micromolecular, lipid-soluble derivatives during their physiological transport.

(d) Design of substrates that intensify the asymmetry in the two-directional operation of a transport system. In the preceding section we began by considering the design of substrates that accelerate the rate-limiting step, so that $V_{\max }$ for transport is increased. Incidental to that effort two other kinds of behavior have been encountered:

(1) Failure of cationic amino acids to be transported as though they are cations, as already noted.

(2) Intensification of the net uphill transport that can be achieved by a given system far above that obtained with any natural substrate.

The reader will note in the last column of Table II that the piperidine amino acids are concentrated much more strongly by the Ehrlich cell than are the ordinary substrates of System $L$ at similar concentrations. Do features of their structures cause these amino acids to respond to an energy gradient across the membrane that is not sensed for example by leucine or isoleucine? By equilibrium dialysis we can detect no evidence of significant binding of 4-amino-1-methylpiperidine-4-carboxylic acid to cell contents after lysis. The difference of electrical potential across the cell membrane can scarcely be sufficient to account for the intensity of its apparent uphill transport, even if we assume that it exists largely as a cation within the cell. The question also arises for 4-amino-1guanylpiperidine-4-carboxylic acid: Why is it concentrated so much more than arginine and lysine, to which it is an analog? Does it sense some energy-releasing flow across the membrane that arginine cannot sense or respond to? Again, no binding to cell contents could be detected after lysis. In any case we must conclude that both systems $L$ and $L \dot{y}$ are capable of asymmetric operation, a capability that has heretofore often been equivocal, especially for System $L \dot{y}$.

A question of parallel interest emerges from comparison of maximal velocities as a function of structure. We have touched in passing on the uniquely high $V_{\max }$ of $\alpha, \gamma$-diaminobutyric acid uptake by System $A .^{31}$ Another diamino acid, S-(2-amino-ethyl)-L-cysteine (thialysine) shows the same paradox of a high maximal velocity of uptake, although in this case for both systems $A$ and $L$ of the Ehrlich cell. This amino acid has a $\mathrm{p} K_{2}^{\prime}$ of $8 \cdot 39,{ }^{32}$ so it should be about $90 \%$ cationic in free solution. It does indeed inhibit the uptake of homoarginine $\left(K_{i} 1.2 \mathrm{mM}\right)$, and a corresponding component of its uptake is inhibitable by homoarginine. This case shows incidentally that ready entry of a diamino acid into System 
$L$ is not necessarily associated with its rejection by System $L \stackrel{+}{y}$, a correlation that might otherwise have raised doubt as to the separateness of the two systems.

Thialysine shows a $K_{m}$ of $20 \mathrm{~mm}$ in its MeAIB-inhibitable (System $A$ ) uptake, and a $V_{\max }$ of about $20 \mathrm{mmol} \mathrm{per} \mathrm{kg}$ cell water $\cdot \mathrm{min}$, about four times the usual value for $V_{\max } \cdot{ }^{23}$ Given that the proton on the distal amino group can be displaced in the environment of the receptor site, the observed reactivity with System $A$ is not surprising because of the linear nature of the thialysine chain. Note that methionine and norleucine are highly reactive substrates for that system as well as System $L$. The $K_{m}$ of thialysine for System $L$ (uptake inhibitable by the norbornane amino acid) is $0.6 \mathrm{~mm}$, and $V_{\max }$ about $6 \mathrm{mmol}$ per $\mathrm{kg}$ cell water $\cdot \mathrm{min}$, the latter about three times the usual values. ${ }^{33}$ Note also, if we correct for the proportion of thialysine present in free solution in forms other than the $\alpha$-zwitterion, that the $K_{m}$ of this amino acid for System $L$ must be unusually low, and hence the quotient describing its rate of uptake at low levels, $V_{\max } / K_{m}$, uniquely high.

Note the following parallelism: $\alpha, \gamma$-Diaminobutyric acid $\mathrm{p} K_{2}^{\prime}=8 \cdot 4$, shows a $V_{\max }$ of not less than ten times typical values for System $A$; thialysine, $\mathrm{p} K_{2}^{\prime}=8 \cdot 39,{ }^{32}$ shows $V_{\max }$ values for both Systems $A$ and $L$ three or four times the usual values. The $\mathrm{p} K_{2}^{\prime}$ value of $8 \cdot 4$ may be almost ideally positioned to permit two effects:

(a) It is low enough so that protonation of the distal amino group may be repressed in an apolar microenvironment at the receptor site.

(b) It may be high enough to permit, subsequently but within the transport process, reprotonation of the distal amino group, which could permit the potential difference across the membrane to accelerate translocation.

This possibility we have tested for by modifying and reversing the potential difference across the plasma membrane of the Ehrlich cell, an effect sought by substituting the non-permeating anion, sulfate, for chloride in the suspending medium. The initial rate of the uptake of thialysine by the neutral amino acid systems is only moderately sensitive to this substitution. The $V_{\max }$ for uptake is, however, still exceptionally high after the chloride content of the medium has been replaced isoosmotically or isotonically with sulfate.

The transport asymmetry noted with some of these diamino acids can be observed also as an asymmetry of the accelerative exchange process, as illustrated in Table IV. Whereas internal BCH greatly stimulates the uptake of cis-1,4-diaminocyclohexanecarboxylic acid, and the latter when presented to the exterior of the cell stimulates correspondingly the exodus of $\mathrm{BCH}$, no such responses are seen in either direction when the cyclohexane amino acid is presented within the cell 
TABLE IV. Representation of sidedness in the mutual acceleration of entry and exodus produced betwcen two amino acids
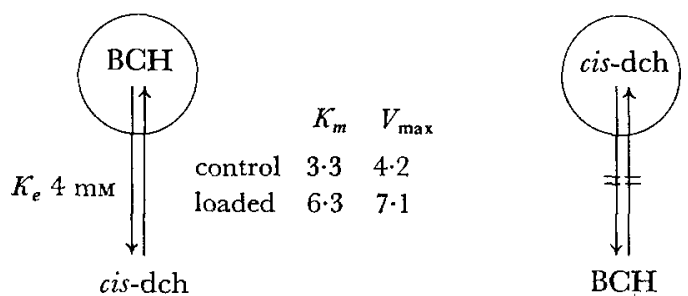

cis-dch
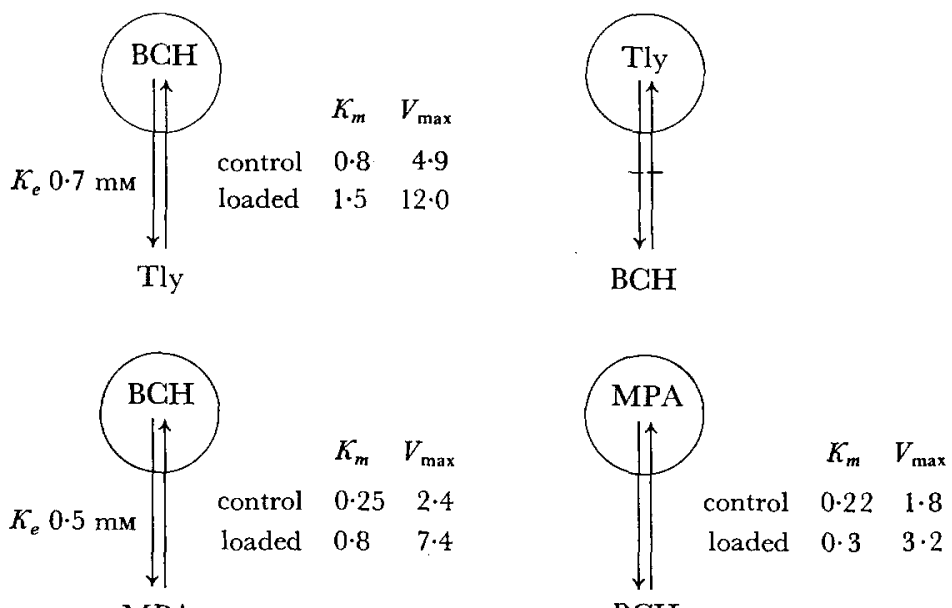

MPA

$\mathrm{BCH}$

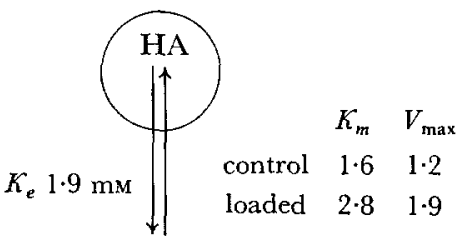

GPA

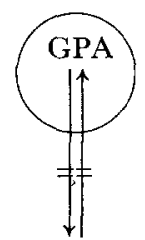

HA

The amino acid enclosed in the circle has been introduced into the cell, in one experiment in labeled, in another in unlabeled form. In the latter case the apparent concentrations were, reading from left to right, line by line, BCH $6 \cdot 2$, dch $7 \cdot 4$, BCH $7 \cdot 1$, Tly $4 \cdot 5, \mathrm{BCH} 7 \cdot 3, \mathrm{MPA}$ 8.4, HA 5.8, GPA 3.0, all in mmoles per kg cell water. The unbarred arrows in the column. at the left show that each of the two amino acids mutually accelerates the migration of the other across the membrane to produce exchange; whereas the barred arrows at the right indicate that when the two amino acids are in the opposite positions, such effects either can not be seen or in the case of thialysine are unusually small. BCH $=2$-aminobicycloheptane-2carboxylic acid; dch = cis-1,4 diaminocyclohexanecarboxylic acid; Tly = L-thialysine; MPA = 4-amino-1-methylpiperidine-4-carboxylic acid; GPA = 4-amino-1-guanylpiperidine-4-carboxylic acid; $\mathrm{HA}=\mathrm{L}$-homoarginine. $K_{e}$ represents the concentration of the external amino acid producing a half-maximal acceleration of exodus of the internal amino acid; $K_{m}$ and $V_{\max }$ describe entry of the external amino acid in the absence and in the presence inside the cell of the other amino acid at the apparent concentrations already stated. Note that the value of $V_{\max } / K_{m}$ was usually not increased by the prior loading; hence acceleration tends to be appreciable only at external substrate levels above $K_{m}$. 
and $\mathrm{BCH}$ on the outside. A similar situation applies to the interaction between $\mathrm{BCH}$ and thialysine: Unusually weak exchange can be seen between internal thialysine and external $\mathrm{BCH}$, whereas exchange is vigorous when the amino acids are presented in the reversed positions.

The behavior presented in these two cases can plausibly be attributed to a relative failure of the internally orientated receptor site to accomplish the paradoxical effect achieved by the externally orientated site for these diamino acids, which we suppose is to stimulate deprotonation of the distal amino group. Since such an effect will cause an accumulation of the diamino acid, it will have an energy cost, presumably invested in maintaining the difference in the state of the site in the two orientations. We face the question whether and how the presumed energy investment is kept smaller when the substrate of System $L$ is instead leucine or phenylalanine, amino acids which are only weakly concentrated by this cell. These results may mean that an intrinsic chemiosmotic responsiveness of the plasma membrane permits transport systems to respond by stimulated energy transduction to substrates that can exist alternatively in charged and uncharged states.

Early in our studies of the heterogeneity of neutral amino acid transport, we were perhaps unduly successful in calling attention to the stronger participation of the $\mathrm{Na}^{+}$-independent System $L$ than of $\mathrm{Na}^{+}$dependent System $A$ in amino acid exchange. Some readers apparently as a result have incorrectly equated $\mathrm{Na}^{+}$independence of uptake with uptake by exchange, and have therefore supposed that the heterogeneity in amino acid uptake can be eliminated by depleting the supply of endogenous amino acids available for exchange. The $\mathrm{Na}^{+}-$ dependent systems are however also exchanging systems. In reticulated and nucleated red cells System $A S C$ is much more sharply restricted to exchange than is System $L$. Our present results show attainment in the absence of added $\mathrm{Na}^{+}$of accumulation of test amino acids in amounts exceeding the initial size of the entire endogenous amino acid pool, and with only modest depletion of that pool. We have for example obtained apparent gradients of more than $32 \mathrm{~mm}$ of 4-amino-1methylpiperidine-4-carboxylic acid, a value exceeding by $30 \%$ the whole content of the endogenous pool, with only $8 \%$ depletion of that pool. The source of energy for this uptake is suggested by a heightened sensitivity of the uptake of 4-amino-1-methylpiperidine-4-carboxylic acid to the presence of 2,4-dinitrophenol or oligomycin. The latter is effective at $0.1 \mu \mathrm{g} / \mathrm{ml}$ in oxygen, but only at 5 or $10 \mu \mathrm{g} / \mathrm{ml}$ in $\mathrm{N}_{2}$ (glucose present). These results are consistent with a utilization of mitochondrial ATP in $\mathrm{O}_{2}$ and of ATP from glycolysis in $\mathrm{N}_{2}$. We can find no basis, however, for any idea that the $\mathrm{Na}^{+}$-dependent: $\mathrm{Na}^{+}$-independent dichotomy in amino acid transport arises from the distinction between net uptake and uptake by exchange. 
We are warned by the third case presented in Table IV that steep asymmetries in the operation of System $L$ do not arise simply from a complete "sidedness" with reference to the exchangeability of two analogs across the plasma membrane. 4-Amino-1-methylpiperidine-4carboxylic acid and $\mathrm{BCH}$ exchange well, no matter which is inside and which is outside; yet the first of these amino acids is concentrated twenty-five times into the Ehrlich cell. The final case tabulated provides a different warning, namely that a difference in the state of protonation of the distal cationic group is not the only way that "sidedness" in exchangeability between two amino acids arises. The exchange between homoarginine and 4-amino-1-guanylpiperidine-4-carboxylic acid is vigorous when homoarginine is the internal member of the pair, but undetectable when the other arginine analog is internal. These compounds both have unequivocally cationic sidechains, and an effective repression of the protonation of the guanidinium group of neither of these seems likely in the biological context. A much more likely explanation is a repression of the protonation of the $\alpha$-amino group of the guanylpiperidine amino acid at the internal face. The positive charge on the nearby guanidine group would facilitate inductively the loss of the proton from the $\alpha$-amino nitrogen. The occurrence of this event could solve another mystery: In the case of the cyclohexane cisdiamino acid, retention of the distal proton might be expected to cause transport via system $L_{y}^{+}$rather than System $L$. If an $\alpha$-proton tends to be lost at the same time that one is gained by the distal amino group, however, the amino acid becomes comparatively unsuitable as a substrate for either system, as actually observed (Table III).

The presence of a distal amino group appears not to be the only structural change that serves to generate an asymmetry between entry into and exodus from the cell. While we were trying several years ago to design an amino acid obstructed to substrate action in all known transport systems, we encountered the strange case of $\alpha, \alpha$-diethylglycine, which is of course an isomer to the leucines. This substance we now recognize as an atypical substrate for System $L$, although at one time its unusual behavior had all but convinced us that it is accumulated instead by a unique transport system. ${ }^{34}$ Diethylglycine is gradually accumulated into the Ehrlich cell by 10 -fold or more, $K_{m}=4 \mathrm{~mm}$, $V_{\max }=2 \cdot 4 \mathrm{mmol}$ per $\mathrm{kg}$ cell water $\cdot \mathrm{min}$. Even though entry is accordingly quite slow, exodus is so extremely slow that high accumulation ultimately results. The two $\alpha$-ethyl groups apparently are barely accommodated by the receptor site for entry, but scarcely if at all by the receptor site for exodus. Hence crowding by the two $\alpha$-ethyl groups presents another structural feature, besides a distal amino group, that brings out more of the asymmetry that must be inherently possible in the bi-directional operation of System $L$.

As for some of the diamino acids already discussed, we see here a logical consequence of the asymmetry of the transport of this amino 
acid: Although external diethylglycine will accelerate the exodus of the norbornane amino acid, and internal norbornane amino acid will accelerate the uptake of diethylglycine, no trans stimulation is seen when the positions of the two amino acids are reversed. An even more crowded analog, $\alpha, \alpha$-dicyclopropylglycine, which shows no saturable uptake by the Ehrlich cell, is nevertheless accumulated by rat brain slices $^{35}$ and by the liver of the intact rat. ${ }^{36}$ Amino acids that serve as characteristic substrates of System $L$ are effective inhibitors of dicyclopropylglycine uptake by these tissues.

Perhaps the most interesting property previously reported for diethylglycine uptake is the near doubling of its initial rate when the $\mathrm{pH}$ is lowered from $\mathrm{pH} 7 \cdot 4$ to $5 .{ }^{34}$ A slowed exodus at $\mathrm{pH} 5$ makes an additional contribution to the higher accumulation at that $\mathrm{pH}$. Hence the asymmetry between the fluxes is much larger at pH 5 than at 7.4.

Basis of structurally determined access to energy sources. Let me attempt a summary of the present section. We have seen that asymmetry between the two fluxes across the plasma membrane, favoring strong accumulation, can be obtained by certain modifications of substrate structure. The most effective modification is the introduction into the sidechain of a distal amino group with a rather low $\mathrm{p} K_{\mathrm{a}}^{\prime}$. This amino group behaves as though it is not protonated when the amino acid enters a neutral amino acid transport system from outside the cell. At the interior surface, however, it fails to show this paradoxical behavior; that is, exodus of these amino acids is exceptionally slow, as though they maintain an unsuitable structure at that surface. Structures of that character should predominate in water solution.

The important feature, however, is that slow exodus is only part of the explanation of the strong accumulation of diamino acids. Their maximal rates of entry are much faster than those of the normal substrates of the neutral amino acid transport systems. Although they behave as though the sidechain were unprotonated on their first combining with the external receptor site, they behave as though the amino group had become protonated by the time they leave the membrane to enter the cytoplasm, not a strange conclusion considering that the internal receptor site apparently sees the free amino acid inside the cell in the less acceptable, $\omega$-protonated form. Furthermore, this presumed transition from a species without net charge to a cationic species must occur during the translocation step, since that step is strongly accelerated by the introduction of the sidechain amino group.

The strong accumulation of these amino acids requires an energy source outside the transport system. That energy demonstrably does not come from pre-existing gradients of exchangeable amino acids, since the high initial velocities of entry are seen even at high substrate levels producing maximal rates, accompanied by relatively small losses of endogenous amino acids. The difference in electrical potential across the membrane appears not to be necessary to the high rate of accumulation. 
For System $A$ we have to think also of the down-gradient flows of the alkali-metal ions, particularly $\mathrm{Na}^{+}$, as energy sources. The stoichiometry of the interdependent or linked fluxes between $\mathrm{Na}^{+}$and diaminobutyric acid into the Ehrlich cell we find if anything lower than the stoichiometry observed for AIB and glycine. Hence at least for that amino acid, the presence of a protonatable, sidechain amino group does not increase the proportional flow of $\mathrm{Na}^{+}$through System $A$. I also see no reason why the presence of this amino group should tighten coupling between the two flows. Furthermore, a deficiency in the energy available from the $\mathrm{Na}^{+}$flow is already a matter of concern even if tight coupling is assumed for ordinary amino acids ${ }^{37}$ (cf. ref. 38). For System $L$ the $\mathrm{Na}^{+}$gradient appears not to be a significant factor, and no energizing gradients except those of amino acids have so far been identified.

What then is the nature of the energy source that is tapped to an unusual extent when an amino group whose protonation is easily modified is present on the amino acid sidechain? All uphill transport must be driven, I believe in a final analysis, by either co-transport or counter-transport, given only that the species entering into linked flux with the transported substrate may be any whatever, even an electron or a proton. This statement I take to follow directly from Curie's theorem, that is, from the circumstance that a completely scalar process cannot drive a vectorial process. Co-transport and counter-transport seem to me to be the modes of linkage between two vectorial processes under ordinary carrier models.

We may then restate the question opening the preceding paragraph as follows: What flow within the membrane is sensed or stimulated to a superior degree when an amino group of suitable $\mathrm{p} K$ is present on the amino acid sidechain? We come readily to the response that the flow may be a flow of the hydrogen ion.

Now the $\mathrm{H}^{+}$gradient across the plasma membrane of the Ehrlich cell has already been examined by use of a weak acid presumed to reach a diffusion-type equilibrium for its protonated form only. ${ }^{39}$ Although, the observed $\mathrm{H}^{+}$gradients vary accordingly to metabolic conditions their magnitudes appear ordinarily even less adequate than the gradients of $\mathrm{Na}^{+}$or $\mathrm{K}^{+}$to account for the accumulation of amino acids.

A provisional interpretation. Because of these observations we suppose that the selected amino acids have a superior ability to establish a vectorial linkage with gradients that are ordinarily to a large degree confined to the membrane somewhat as suggested by Williams, ${ }^{40}$ and not fully expressed between the two aqueous phases separated by the membrane. It is perhaps somewhat arbitrary whether we describe this gradient as a gradient of hydrogen ion or as a gradient of polarization of molecular structure, somewhat like proposals made recently by Green and $\mathrm{Ji}^{41}$ for the inner mitochondrial membrane. 
This gradient is located we suppose in patterns of protein structure that extend here and there almost all the way through the barrier material of the membrane. This gradient may be represented as a gradient in the degree of protonation of otherwise similar protonaccepting groups of protein structure; or it may be represented as gradients of electron distribution set up by electron flows. Its effects can, however, be communicated to the composition of the two adjoining aqueous phases to variable degrees through the presence of certain substrates that fit certain corresponding receptor sites. Some of these receptor sites recognize ATP and $\mathrm{Na}^{+}$, others recognize $\mathrm{K}^{+}$, still others certain amino acids alone, or certain amino acids along with $\mathrm{Na}^{+}$or $\mathrm{H}^{+}$. A cation presented in the apolar screening environment of an ionophore may establish a superior communication. In still other cases sugars along with $\mathrm{Na}^{+}$are recognized. The consequence is that the gradient within the membrane is caused to do work or to have work done on it, although the responses of the substrate do not necessarily measure the full intensity of the standing gradient, the coupling being loose, and varying according to substrate structure. The membrane responds to the stimulated energy transduction caused by transported substrates, by increasing the fundamental flow that maintains its polarization. For membranes containing an electron transport chain, the fundamental flow that maintains polarization may be one of electrons from a donor, through the structure on which the gradient is impressed, to a receptor; for others it may be the reversal of a chargeseparating step produced by the catalytic hydrolysis of ATP.

Mediated transport systems may transduce variable amounts of energy via the standing gradient of the membrane of the metabolizing cell, depending among other factors on the comparative filling by the substrate of the corresponding receptor sites at the two membrane surfaces. The consequence of the presence of a gradient of polarization within the membrane is that the two receptor sites appear to a selected substrate to be dissimilar, and to respond to the substrate differently by conformational change, leading to an energy-utilizing asymmetry of fluxes. Thialysine, for example, reacts with the external receptor site for neutral amino acids as though its sidechain had entered a region in which the chemical potential of the hydrogen ion corresponds, so to speak, to a $\mathrm{pH}$ as much as two units above that of the external medium. At the internal surface of the membrane, no such paradox is apparent; perhaps a $\mathrm{H}^{+}$-deficiency will be found instead.

Influence of $\mathrm{H}^{+}$on amino acid transport. If the above interpretation is correct, then it would be strange if the hydrogen ion distribution had no influence whatever on amino acid transport. Uptake by the $\mathrm{Na}^{+}$dependent systems is in fact greatly slowed by lowering the $\mathrm{pH}$ from $7 \cdot 4$ to 5 , although we have attributed this effect to a competition of $\mathrm{H}^{+}$ for the site at which the essential $\mathrm{Na}^{+}$binds. In contrast the uptake of 
such amino acids as leucine and phenylalanine is decreasing by only 10 to $20 \%$ by the same $\mathrm{pH}$ lowering. ${ }^{33}$

Our earlier observation that $\alpha, \alpha$-diethylglycine uptake is stimulated by lowering the $\mathrm{pH}^{34}$ finally brought us to see that this response is characteristic for System $L$. We now appreciate that this amino acid serves as a marginally suitable substrate for entry (although scarcely for exodus) by that system. The norbornane amino acid also proves to have its uptake strongly accelerated and its exodus slowed when the $\mathrm{pH}$ of the suspending medium is lowered. If our observations are made in media in which choline replaces $\mathrm{Na}^{+}$, the uptake of leucine, isoleucine and valine shows similar acceleration by external $\mathrm{H}^{+}$.

Eddy and Nowacki have recently shown that yeast cells take up $\mathrm{H}^{+}$ and lose $\mathrm{K}^{+}$when such amino acids as glycine, phenylalanine and methionine are taken up. These authors have proposed that these ion movements may bear the same relationship as the correspondingly opposed movements of $\mathrm{Na}^{+}$and $\mathrm{K}^{+}$in animal cells, which they show may well drive amino acid uptake by ascites cells. ${ }^{28}$ The cation gradients across the plasma membrane may in neither case, however, be essential intermediates in the transduction between metabolic energy and osmotic energy. The actual intermediating gradients may as described above lie within the membrane, reflected only incompletely and unreliably by gradients and flows of cations between the two aqueous solutions.

Recent experiments by Gunnar Ronquist in this laboratory indicate that the accumulation of $\alpha$-aminoisobutyric acid and sarcosine by the Ehrlich cell can be largely dissociated from $\mathrm{Na}^{+}$influx. This effect has been obtained by incubating the cells twice for $5 \mathrm{~min}$ each time in the presence of AIB at $10 \mathrm{~mm}$, with $\mathrm{Na}^{+}$at $15 \mathrm{~mm}$, for the purpose of extensively replacing the endogenous amino acids with AIB. The cells come as a result to contain about 15 to $18 \mathrm{~mm} \mathrm{AIB}$ and about 8 to 10 mM of all other amino acids.

We observe that at $\left[{ }^{22} \mathrm{Na}^{+}\right]_{0}=120 \mathrm{mv}$ cells treated thus will take up large further quantities of AIB or sarcosine to high gradients with only a very small associated uptake of $\mathrm{Na}^{+}$. Uptake is however small from $\mathrm{Na}^{+}$-free, choline-containing medium. The uptake of methionine and alanine is still accompanied by approximately stoichiometric uptake of $\mathrm{Na}^{+}$. Apparently then the structures of the amino acids present on the two sides of the membrane can determine the extent to which transport is linked with $\mathrm{Na}^{+}$movements in System $A$ as well as in System $A S C$.

If neither $\mathrm{Na}^{+}$or $\mathrm{H}^{+}$are obligatory co-substrates for uptake of amino acids by the Ehrlich cell, then the gradients of neither of these ions between the two aqueous solutions seem likely to be obligatory mediating stages in the coupled flow of energy. On the other hand, energy from the $\mathrm{Na}^{+}$gradient appears to be available under normal conditions to drive the uptake of certain amino acids, and energy from the $\mathrm{H}^{+}$ 
gradient may be available for other systems or in other cells for the same purpose. As we wrote in 1962, "Uphill transports may well not be placed in series, e.g., with alkali-metal transport driving amino acid transport; but in parallel, with many or all uphill transports driven by the response of the membrane to ATP cleavage [or we will now add, an electron or proton flow]. The maintenance of an activated, transportresponsive state of the membrane may then be aided by the presence of high cellular levels of potassium ion or of amino acids," i.e., of gradients already generated by transport. ${ }^{42}$ This seems now provisionally the most plausible form of the alkali-metal gradient hypothesis.

The foregoing treatment will I hope help to show that the study of transport changes produced by modifications of substrate structure is a biochemical as well as a physiological approach to transport. The approach has undoubtedly suffered occasionally from too purely a biological interest (e.g., which of the ordinary sugars or amino acids are transported, which are not?) with perhaps insufficient attention to the meaning of the structural differences among the several analogous substrates transported by a given system.

An important feature brought out by the foregoing results is that sensing groups or probes can be introduced into transport substrates to report the location of either specific structures or special microenvironments encountered during the sequential events of biological transport. The variation permissible in substrate structure is large enough for the insertion of chemical groups that may report nearness to a specific inorganic ion or other structure, or the degree of polarity of the environments encountered by a given part of the substrate structure. Other sensing groups that might be introduced include fluorescing structures or paramagnetic structures from which signals could then be obtained. The surprising amount of space available, particularly for sidechain structures of amino acids by transport System $L$, make possible a very wide range of tests. Similar possibilities appear to exist for transport systems for other metabolites. Room is presumably also available for introduction of some types of pharmacologically active structures into these regions of transport substrates.

6. Similar goals for recognition sites serving for the transmission of information. Finally, I should like to point out that objectives similar to those discussed so far make important the study of substrate structure on membrane-borne recognition sites of other kinds, which likewise do not apparently call for the destabilization of the substrate. I refer particularly to receptor sites by which cells can detect the presence in their external environments of gradients of nutrients and respond by chemotaxis ${ }^{43,44}$ or sites by which they can maintain a given degree of induction of a transport system,${ }^{45}$ or prevent a spontaneous intensification of transport on incubation of the embryonic chick heart in vitro ${ }^{46}$ or by which they detect elevated levels of a metabolite in their 
environment, and respond for example by releasing a hormone, e.g. insulin or glucagon. ${ }^{47-49}$ Receptor sites on neurons of the central nervous system may account for the potent stimulatory and inhibitory effects of several amino acids when released into the synaptic area. ${ }^{35} \mathrm{~A}$ broad range of physiological functions obviously is served by receptor sites of such types. Some sites of these kinds may have an internal location, rather than on the plasma membrane; in other cases the location is unknown.

One kind of site with an internal rather than an external location has been proposed whereby an accumulated amino acid may cause a trans inhibition of further uptake of that amino acid, or indeed of other substrates of the same system. In Streptomyces hydrogenans a different specificity pattern has been shown for trans inhibition than for uptake per se. ${ }^{50}$ Hence a separate site is believed to activate a loop for feedback inhibition of further uptake. In yeast a separate site is also believed to serve, although the evidence may not be decisive, ${ }^{51}$ whereas in Neurospora the phenomenon is not believed to entail a distinct site, as discussed under Item 4 above, since the specificity for uptake and that for producing trans inhibition are indistinguishable. ${ }^{52} \mathrm{It}$ is conceivable that the structural specificity for signals to arise from substrate passage through a transport system may be narrower than the specificity for transport per se. One can imagine that some of the substrates of a given transport system might fail to elicit a signal arising say from the generation of the kind of new membrane asymmetry implied by trans stimulation simply because these substrates are translocated too slowly to cause any such new asymmetry. Hence the structural requirement for signalling an elevated metabolite level by a given transport system may be somewhat more restrictive than those for transport by it. The discrepancy noted in $S$. hydrogenans is, however, in the opposite direction; hence it is unlikely that the transport site per se is the source of the signal in that special case.

These categories of receptor sites show some of the characteristics of transport sites, including the absence of any necessary destabilizing of the provoking agent. In the case of chemotaxis, the same binding proteins may serve for both material and informational transport. ${ }^{44}$ These various sites may well have common evolutionary or differentiative origins; in some instances they may serve concurrently for transport and for information transfer. The opportunity to design analogs that inhibit but do not cause the response in question may permit separate study of the filling of the recognition site on the one hand, and, on the other hand, the sending either of material or of a signal to the cell interior.

\section{References}

1. T. Z. Csáky, Ber. ges., 108 (1938) 620; Z. Physiol. Chem., 227 (1942) 47.

2. C. G. Mark and A. H. Romano, Biochim. Biophys. Acta, 249 (1971) 216. 
3. H. S. Tager and H. N. Christensen, J. Biol. Chem., 246 (1971) 7572.

4. P. Mitchell and J. Moyle, Nature, 182 (1958) 372.

5. G. Gachelin, Eur. J. Biochem., 16 (1970) 342.

6. L. Lieve and B. D. Davis, J. Biol. Chem., 240 (1965) 4362.

7. E. A. Berger, J. H. Weiner and L. A. Heppel, Federation Proceedings, 30 (1971) 1061.

8. H. N. Christensen, Advances in Enzymology, 32 (1969) 1.

9. H. N. Christensen, M. E. Handlogten, I. Lam, H. S. Tager and R. Zand, J. Biol. Chem., 244 (1969) 1510.

10. G. E. Furlong and J. H. Weiner, Biochem. Biophys. Res. Comm., 38 (1970) 1076.

11. E. L. Thomas, T.-C. Shao and H. N. Christensen, J. Biol. Chem., 246 (1971) 1677.

12. H. N. Christensen and M. E. Handlogten, FEBS Letters, 3 (1969) 14.

13. H. N. Christensen, M. E. Handlogten and E. L. Thomas, Proc. Nat. Acad. Sci. U.S., 63 (1969) 948

14. E. L. Thomas and H. N. Christensen, J. Biol. Chem., 246 (1971) 1682.

15. J. A. Antonioli and H. N. Christensen, J. Biol. Chem., 244 (1969) 1505.

16. B. H. Koser and H. N. Christensen, Biochim. Biophys. Acta, 241 (1971) 9.

17. E. L. Thomas and H. N. Christensen, Biochem. Biophys. Res. Comm., 40 (1970) 277.

18. P. G. Le Fevre and J. K. Marshall, Am. J. Physiol., 194 (1959) 333.

19. A. Kleinzeller, Biachim. Biophys. Acta, 211 (1970) 264.

20. H. N. Christensen, E. L. Thomas and M. E. Handlogten, Biochim. Biophys. Acta, 193 (1969) 228.

21. W. F. Caspary, N. R. Stevenson and R. K. Crane, Biochim. Biophys. Acta, 193 (1969) 168.

22. P. F. Baker and S. J. Potashner, Biochim. Biophys. Acta, 249 (1971) 616.

23. Y. Inui and H. N. Christensen, J. Gen. Physiol., 50 (1966) 203.

24. H. N. Christensen, in Roles of Membranes in Secretory Processes, Proceedings of an International Conference on Biological Membranes, Italy, 1971, North Holland Publ. Co., 1972.

25. E. Heinz and R. P. Durbin, J. Gen. Physiol., 41 (1957) 101.

26. M. L. Pall, Biochim. Biophys. Acta, 233 (1971) 201.

27. D. L. Oxender and B. Whitmore, Fed. Proc., 25 (1966) 592.

28. A. A. Eddy and J. A. Nowacki, Biochem. J., 122 (1971) $70 \mathrm{~L}$.

29. H. N. Christensen, M. E. Handlogten and A. M. Cullen, Federation Proceedings, 30 (1971) 1116.

30. E. Fischer and G. Zemplen, Ber. d. chem. ges., 42 (1909) 4878.

31. H. N. Christensen and M. Liang, J. Biol. Chem., 241 (1966) 5542.

32. L. Eldjarn, Scan. J. Clin. Lat. Inv., 6, Suppl. 13 (1966) 1.

33. D. L. Oxender and H. N. Christensen, J. Biol. Chem., 238 (1963) 3686.

34. H. N. Christensen and M. Liang, J. Biol. Chem., 240 (1965) 3601.

35. H. N. Christensen, in: "Role of Vitamin $B_{6}$ in Neurology," E. Ebadi (eds.), Advances in Biochem. Pharmacol., 4 (1972) 39.

36. H. N. Christensen and A. M. Cullen, Biochim. Biophys. Acta, 150 (1968) 237.

37. J. A. Schafer and E. Heinz, Biochim. Biophys. Acta, 249 (1971) 15.

38. E. Heinz (ed.), Proceedings of Symposium on Coupling Between Electrolyte and Non-Electrolyte Transport in Cells, Erbach, 1971, North Holland Publishing Company, 1972.

39. D. J. Poole, T. L. Butler and W. J. Waddell, J. Nat. Cancer Inst., 32 (1964) 939.

40. R.J. P. Williams, 7. Theoret. Biol., 1 (1961) 1.

41. D. E. Green and S.Ji, Proc. Nat. Acad. Sci. U.S., 69 (1972) 726.

42. H. N. Christensen, Biological Transport, W. J. Benjamin, New York, 1962, pp. 86-87.

43. J. Adler, Science, 166 (1969) 1588.

44. J. Adler and G. L. Hazelbauer, Nature Ner Biology, 230 (1971) 101.

45. H. H. Winkler, J. Bact., 101 (1971) 470: 107 (1971) 74.

46. G. D. Gazzola, R. Franchi, V. Saibene, P. Ronchi and G. G. Guidotta, Biochim. Biophys. Acta, 266 (1972) 407.

47. H. N. Christensen and A. M. Cullen, J. Biol. Chem., 244 (1969) 1521.

48. S. S. Fajans, R. Quibrera, S. Pek, J. C. Floyd, Jr. and H. N. Christensen, 7. Clin. Endocrinol. Metab., 33 (1971) 35.

49. H. N. Christensen, B. Hellman, Å. Lernmark, J. Sehlin, H. S. Tager and I.-B. Täljedal, Biochim. Biophys. Acta, 241 (1971) 341.

50. K. Ring, W. Gross and E. Heinz, Arch. Biochem. Biophys., 137 (1970) 243.

51. M. Grenson, M. Crabeel, J. M. Wiame and J. Bechet, Biochim. Biophys. Acta, 30 (1968) 414 .

52. M. L. Pall and K. A. Kelly, Biochem. Biophys. Res. Comm., 42 (1971) 940. 